\title{
EP NEWS AND INNOVATIONS
}

\section{Innovative Device Could Offer New Hope for Heart Patients}

A UConn graduate student is developing a new micro-scale power source that is significantly smaller and more efficient than the batteries currently used in most cardiac pacemakers today. The student is developing an implantable electronic device that draws its power from an ultra-small, ultra-thin supercapacitor.

Implantable cardiac pacemakers have extended the lives of millions of people since they were first introduced in the late 1950s. But current models are not without their drawbacks. They are powered by bulky batteries that require surgery when their charge weakens, and they need to be replaced. This poses a risk for infection. The batteries' internal electronic components also can be toxic to the body should they ever leak.

About the size of a postage stamp, the power source is bendable and looks like a thin piece of film. In lab tests, the device has also been shown to be non-toxic to living cells. This feature is possible because the device uses the patient's own blood serum as an electrolyte rather than draw its power from a potentially toxic lithium-ion battery, which is the preferred power source for most cardiac pacemakers today.

The power system's efficiency allows it to maintain a charge for a long period of time, a feature that could make it suitable for other bioelectronic devices such as implantable neurostimulators, which are used to treat some patients with Parkinson's disease.

Exactly how the power system works and how a patient's biofluids serve as an electrolyte is still proprietary. The research team expects more details to be released soon once their research paper is accepted for publication.

\section{Smallest single lead medical grade ECG, Kardia Band}

The availability of Kardia Band, the world's smallest single lead medical grade ECG has launched. Kardia Band works with Apple Watch to provide a single lead medical grade ECG of the user's heart rhythm to identify possible Atrial Fibrillation - a leading cause of AF-related stroke in the UK.

People over 40 have a one in four lifetime risk of developing Atrial Fibrillation, which leads to a 500 per cent increased risk of AF-related stroke. Despite this, research commissioned of 2,000 UK adults found that only 9 per cent identified it as a cause of stroke, with a further 24 per cent saying stroke can't be prevented and 7 percent believing stroke is just 'bad luck'.

100,000 people in the UK die annually because of heart rhythm disorders, even though 80 per cent of deaths could be prevented. Only 16 per cent of Britons questioned agreed that monitoring heart rhythm can help prevent AF-related stroke.

The Kardia Band measures heart rhythm with a quick touch of the watch strap and can reveal instantly if it is normal or irregular. It operates directly with Apple Watch via the Kardia app (downloadable from Apple App Store) and can immediately sync this heart rhythm analysis with other devices such as an iPad or iPhone. Results can also be directly shared with a healthcare professional or person's circle of care.

Kardia technology has the potential to save hundreds of lives a year and the mobile version, Kardia Mobile, was shown as an example of MedTech innovation by the Head of NHS England, Simon Stevens at the NHS Confederation 2016.

\section{Launch of Interface between Niobe ${ }^{\circledR}$ ES System and Philips' State-of-the-art X-ray System}

With innovative robotic technologies for the treatment of cardiac arrhythmias, there has been an announcement of the release of an interface between its remote magnetic navigation system for electrophysiology (EP) procedures and the Allura Xper FD10 cardiovascular x-ray system. The interface allows interoperability of the Niobe ${ }^{\circledR}$ system with the Allura Xper FD10 version R8.2 system and is available worldwide.

The Allura Xper FD10 is a ceiling-suspended imaging system widely used for EP and other cardiac interventions. The system offers rotational scan for high-resolution 3D images of cardiac anatomy in real-time, managed x-ray, and storage and retrieval of multi-modality cardiology information. 
The 1st Revivent $\mathrm{TC}^{\mathrm{TM}}$ TransCatheter Ventricular Enhancement procedure for Ischemic Cardiomyopathy treatment in the Netherlands

The announcement of the first clinical use in the Netherlands of its closed-chest Revivent $\mathrm{TC}^{\mathrm{TM}}$ TransCatheter Ventricular Enhancement System. The Less Invasive Ventricular Enhancement ${ }^{\mathrm{TM}}$ or LIVE ${ }^{\mathrm{TM}}$ procedure was performed in Nieuwegein, The Netherlands.

The device was successfully implanted with four micro-anchor pairs in a patient suffering from ischemic heart failure. The procedure reshaped the left ventricle and decreased the Left Ventricular End Systolic Volume Index (LVESVI). Left Ventricular volume reduction is a common measure of cardiac performance that significantly impacts the short and long-term survival rates. By remodeling the LV to a more normal shape and size, the implant improves pumping efficiency, decreases wall stress, and immediately reverses patient symptoms.

The company recently received CE mark certification for its closed-chest Revivent TC TransCatheter Ventricular Enhancement System for plication of scar tissue in post-MI, ischemic cardiomyopathy patients. Placement of the Revivent TC System via the LIVE procedure obviates the need for more invasive surgery. Instead, small titanium anchors are placed along the outer surface of the heart and along one of the interior walls via a catheter-based approach. The anchors are then pulled towards one another, effectively excluding the scarred and non-functioning heart wall. Ventricular volume is immediately reduced as a result of the exclusion, by as much as 30-40 percent1.

The Revivent TC System is approved for sale in Europe; it is not approved for sale in the United States.

\section{UK's first heart operation uses new system to analyze electrical activity during AF}

The UK's first heart operations using a novel software platform to pinpoint the source of the heart condition have been carried out in Leicester.

The patients suffered from a condition known as atrial fibrillation (AF) -- the commonest heart rhythm disturbance affecting more than 1 million people in the UK.

All three patients have now returned home following the operations which were completed successfully.

$\mathrm{AF}$ is a condition that causes the upper chambers of the heart (atria) to beat very fast and irregularly due to chaotic electrical activity. As a result the atria do not beat in an organized way and pump less efficiently, increasing the likelihood of stroke and heart failure.

Initial treatment for $\mathrm{AF}$ is with medication to control the heart rate or reduce $\mathrm{AF}$ episodes using drugs. In many patients, AF fails to be controlled by medication and continues to cause debilitating symptoms.

Catheter ablation has been increasingly used in patients with AF over the past decade or so. The procedure involves inserting electrical wires called catheters into the heart to ablate (or 'burn') the abnormal areas in the heart that are causing or sustaining AF. In patients who are in an early phase of the condition, this procedure has been shown to be reasonably effective requiring limited burning in well-defined heart regions (e.g. pulmonary veins).

However, the results of AF ablation in patients with more advanced form of the condition is less than ideal with variable results and patients often need to undergo several procedures with suboptimal long term outcome. The conventional approach involves extensive ablation on many different atrial locations due to the seemingly chaotic electrical activity seen. More recent data support the presence of focal or rotational sources (or rotors) which may be driving the chaotic process.

The new software platform developed to "decode" the chaotic electrical signals and represent the activity in the form of rotor maps - allow researchers to see the rotors and the centers of rotation analogous. The location of these rotors are different in different patients and hence this new software platform allows a personalized or precision approach to target localized sources for ablation rather than having to ablate over a wide area in the atrial chambers. The initial results from studies conducted in USA and some European centers i.e. Germany are promising and suggest better efficacy than the conventional approach with extensive ablation.

The map generated with the new system is quite unique and there is much research to be done to fully understand the different behavior of these maps in different patients. Having access to this new software algorithm allows researchers to examine the response to $\mathrm{AF}$ ablation using this approach in patients first hand. 
AF ablation in patients with more advanced form of the condition is challenging using the current approach, often needing extensive ablation.

This new software platform uses mathematical processing techniques to 'decode' the chaotic behavior to reveal the underlying focal or rotational activity which are believed to be the driver of the rhythm disturbance. Hence it is hoped that targeting these localized critical circuits would lead to better results and less ablation required which should be translated to better patient outcome.

The variable results from the current approach to $\mathrm{AF}$ ablation highlights that there is a significant component of individual difference between patients which needs a personalized or precision medicine approach to identify the unique characteristics in the particular patient that researchers are treating.

\section{Lifevest Wearable Defibrillator Proven Safe and Effective in Largest German Registry}

Results from the largest German registry of patients prescribed the LifeVest ${ }^{\circledR}$ Wearable Defibrillator reaffirm that the LifeVest is a safe and effective therapy for the prevention of sudden cardiac death (SCD) in the German healthcare system.

The study evaluated more than 6,000 patients from 404 clinical centers across all 16 German states, representing the largest LifeVest patient registry in Europe. Investigators demonstrated that risk for SCD was high in the early period following an acute cardiac event. Seventy percent (70\%) of treated patients received therapy in the first 40 days and $89 \%$ in the first 90 days of LifeVest use. Treated patients demonstrated a $93 \%$ event survival. In addition, overall survival among all patients prescribed the LifeVest was $99.9 \%$ at the end of LifeVest use.

The wearable defibrillator is included in the 2015 European Society of Cardiology Guidelines for the Management of Patients with Ventricular Arrhythmias and the Prevention of Sudden Cardiac Death and has received formal recommendations in the 2016 American Heart Association Science Advisory for the Wearable Cardioverter Defibrillator.

Tens of thousands of people around the world are protected from sudden cardiac death by the LifeVest. The LifeVest is used for a wide range of patient conditions or situations, including following a myocardial infarction, before or after coronary revascularization, and for those with cardiomyopathy or congestive heart failure that places them at particular risk. The LifeVest gives physicians time to optimize medical therapy and assess a patient's long-term risk for sudden death.

The LifeVest is lightweight and easy to wear, allowing patients to return to their common activities of daily living, while having the peace of mind that they are protected from SCD. The LifeVest continuously monitors the patient's heart and, if a life-threatening heart rhythm is detected, the device delivers a treatment shock to restore normal heart rhythm.

\section{Better Prognosis for Some PCI Patients When Operators Perform More Procedures}

Volume matters when it comes to who does certain procedures to removing heart-attack causing blockages from arteries.

Researchers in China found patients undergoing left main coronary artery percutaneous coronary intervention (PCI) had better outcomes when the procedure was performed by experienced, high-volume operators-even though the patients treated by the experienced operators tended to have more complex and extensive heart disease. Left-main PCI can be used to treat patients with advanced coronary artery disease. In this procedure, a drug-eluting stent is placed in the left main coronary artery to open the blockage. The technique is minimally invasive and has been associated with fewer complications, short hospital stays, and quicker recovery time than coronary artery bypass graft (CABG) surgery.

The researchers examined the records of 1,948 patients who underwent left-main PCI between January 2004 and December 2011, with 25 operators performing the procedure. Of those patients, 1,422 were treated by seven experienced, high-volume operators and 526 patients were treated by 18 less experienced, low-volume operators. High-volume operators performed a mean of 25 left-main PCIs per year, while low-volume operators performed a mean of 4 left-main PCIs per year.

Patients treated by high-volume operators had better short- and long-term outcomes. Death within 30 days was 0.6 percent with high-volume operators and 2.1 percent with low-volume operators. Patients of high-volume operators also had lower risks of cardiac death and all-cause mortality, as well as a trend toward a decreased risk of stent thrombosis. 
After three years, patients treated by experienced, high-volume operators experienced a significantly lower risk of cardiac death.

These trends were observed even though patients who underwent left-main PCI with a high-volume operator had more extensive coronary artery disease and underwent more technically complex procedures. High-volume operators were also more likely to use first-generation, rather than superior second-generation, drug-eluting stents.

This is the first study to conclusively demonstrate that operator experience impacts the prognosis of patients who undergo high-risk PCI.

In an editorial comment accompanying the study, examined data from the ACC's National Cardiovascular Data Registry's (NCDR) CathPCI Registry to determine the amount of left-main PCI's performed by high- and low-volume operators in the United States. They found that for left-main PCI's performed between April 2015 and March 2016, only 1 percent of operators would be considered high-volume based on the study's criteria of having performed at least 15 left-main PCI's per year for at least three consecutive years. Additionally, these operators performed only 8.4 percent of these annual procedures in the United States.

\section{Analysis Shows Positive Association Between Cardiac Resynchronization Therapy and Patients' Increased Adherence to Heart Failure Medication Regimens}

Analysis reveals patients increasingly adhere to heart failure medications after they receive cardiac resynchronization therapy (CRT) devices. The analysis of more than 4,500 patients revealed that compliance with their medications increased 67 percent 24 months after receiving their CRT implants, compared to the 24 months prior to implant $(p<0.001)$. The results were presented at the 2016 Heart Failure Society of America (HFSA) Scientific Meeting.

CRT is a proven treatment for select patients with heart failure, and in combination with optimal medical therapy has been shown to further reduce patients' symptoms and extend their survival. Patients' adherence to medication is a major component of heart failure self-management, and commonly prescribed therapies include angiotensinconverting enzyme inhibitors (ACE-I) or angiotensin receptor blockers (ARB), beta-blockers (BB), direct renin inhibitors (DRI), and diuretics for patients with heart failure and a reduced ejection fraction. Additionally, for heart failure patients whose hearts pump out of sync, professional medical guidelines recommend cardiac resynchronization therapy-pacemakers (CRT-P) or -defibrillators (CRT-D).

The retrospective analysis used administrative claims data from 4,512 patients, younger than 65 years old, who received CRT devices between January 2008 and December 2014. Patients' adherence to a regimen of guidelinerecommended medication classes (diuretic, ACE-I, ARB, BB, aldosterone antagonist, digoxin, and statin) before CRT and after CRT implantation was assessed using proportion of days covered (PDC), which measures the availability of all prescribed medications simultaneously in these patients' homes.

The number of patients considered fully compliant with their medication regimens (PDC $>=80$ percent) nearly doubled at 12 months following CRT implants. Additionally, the proportion of compliant patients increased substantially for individual medication classes at 12 months following CRT implants (ACE-I at 54.4 percent vs 37.1 percent, BB at 58.2 percent vs 33.9 percent, loop diuretic at 41.8 percent vs 27 percent).

In collaboration with leading clinicians, researchers and scientists worldwide, the company offers the broadest range of innovative medical technology for the interventional and surgical treatment of cardiovascular disease and cardiac arrhythmias. The company strives to offer products and services of the highest quality that deliver clinical and economic value to healthcare consumers and providers around the world.

\section{World's Smallest Minimally Invasive Pacemaker}

Known as the world's smallest pacemaker, the one-inch Micra TPS, which is 93 percent smaller than conventional pacemakers and smaller than a AA battery, is implanted directly into the patient's right ventricle through a vein in the leg. It is a single-chamber pacing system that paces only the right ventricle of the heart.

In the past, single chamber pacemakers were implanted under the skin near the collarbone and had wired leads that ran through a vein directly to the heart's right ventricle, creating a "pocket" under the skin. Unlike these conventional pacemakers, the Micra TPS is self-contained and does not have wired leads. It latches directly onto the heart using small hooks and can be repositioned if needed. 
Although every patient needing a pacemaker is not a candidate for the Micra TPS, it can be of great benefit to those who are. Patients will not have a chest scar or a bump, meaning they will not have the discomfort or visible, physical reminder of a pacemaker under the skin.

The FDA approved the Micra TPS in April.

The tiny pacemaker is used to treat bradycardia, a condition characterized by a slow or irregular heart rhythm, usually fewer than 60 beats per minute (normal heart rate is $60-100 \mathrm{bpm}$ ). At this rate, the heart is unable to pump enough oxygen-rich blood to the body during normal activity or exercise, causing patients to experience dizziness, fatigue, shortness of breath or fainting spells.

Pacemakers, the most common way to treat bradycardia, help restore the heart's normal rhythm and relieve symptoms by sending electrical impulses to the heart to increase the heart rate. The pacemaker is implanted into one chamber of the heart, the right ventricle.

\section{Ultra-Portable Device Connects Patients with Physicians and Provides Daily Data Transmissions}

CardioMessenger Smart is a portable monitoring device, about the size of a modern smartphone, that keeps pacemaker, implantable cardioverter defibrillator (ICD), and insertable cardiac monitor (ICM) patients connected to their physician remotely, enabling more efficient care management-anywhere in the world.

CardioMessenger Smart provides secure, fully automatic transmission of vital information from a patient's cardiac implant to their physician via home monitoring. This includes daily, automatic reports and fully customizable alerts that can be programmed to the physician's specifications. As demonstrated by the TRUST1, COMPAS2 and IN-TIME3clinical studies, Home Monitoring can significantly reduce hospitalization, stroke and mortality. CardioMessenger Smart recently received Food and Drug Administration approval.

The portability of CardioMessenger Smart helps ensure patient compliance and the consistent transmission of data necessary for physicians to identify and prevent potential cardiac events. CardioMessenger Smart is fully automatic, providing daily reports of cardiac activity via worldwide cellular networks to physicians without intervention from the patient. ${ }^{*}$ CAUTION - Investigational device. Limited by United States law to investigational use.

GARFIELD-AF Registry Exceeds its 57,000 Enrolment Target, New Results Show Superior Performance of the GARFIELD-AF Score, a Novel Machine Learning Tool, in Predicting Ischemic Stroke in Low-Risk Patients

Global data from GARFIELD-AF provides real world insights on managing atrial fibrillation (AF) in everyday practice

GARFIELD-AF is the largest ongoing prospective AF registry and enrolment is now complete, with 57,262 patients recruited across five cohorts.

New analyses from the Global Anticoagulant Registry in the FIELD - Atrial Fibrillation (GARFIELD-AF) show that a novel computer-generated machine learning risk model - the GARFIELD-AF Score - is superior to CHA2DS2-VASc in predicting all-cause mortality, ischaemic stroke/systemic embolism (SE) and major bleeding in low-risk patients. A simplified GARFIELD-AF Score, potentially suitable for web applications, has been developed and its performance assessed using an independent contemporary registry from the USA, ORBIT-AF.

The GARFIELD-AF Score was developed after contemporary data from the GARFIELD-AF Registry revealed that approximately half of low-risk patients are receiving anticoagulation treatment.

The GARFIELD-AF Score is based on the analyses of data from 38,984 patients, enrolled in GARFIELD-AF betweenMarch 2010 and July 2015. In low-risk patients, the GARFIELD-AF Score offered superior discriminatory value in predicting all-cause mortality, ischaemic stroke/SE or haemorrhagic stroke/major bleed with C statistics* ${ }^{*}$ : 0.72 , 0.62 and 0.72 , respectively, compared with CHA2DS2-VASc which had C statistics of: $0.56,0.56$ and 0.57 , respectively for each endpoint.

${ }^{*} \mathrm{~A}$ higher $\mathrm{C}$ statistic demonstrates higher accuracy at predicting outcomes

Further insights from GARFIELD-AF showed a shift in how AF is being managed across the globe. The number of patients receiving anticoagulant treatment for stroke prevention has increased substantially from $57 \%$ to $71 \%$ between March 2010 and August 2015. This shift in anticoagulant management of patients with AF is largely due to the marked increase in non-vitamin $\mathrm{K}$ antagonist oral anticoagulants (NOACs) with or without an antiplatelet prescribing from 
$4.1 \%$ to $37.0 \%$, with a corresponding fall in vitamin $\mathrm{K}$ antagonists (VKAs) and antiplatelet (combined or alone) use from $83.4 \%$ to $50.6 \%$.

New data, showcased during the GARFIELD-AF Satellite Symposium at ESC Congress 2016, revealed that mortality rates are higher during the first month after diagnosis of $\mathrm{AF}$ than at any other time over the subsequent 2-year followup. The risk of early death is greater in patients with prior history of myocardial infarction/unstable angina, moderateto-severe chronic kidney disease, or stroke than in those without these comorbidities.

2016 marks the end of the enrolment phase for GARFIELD-AF, with 57,262 patients now enrolled. GARFIELD-AF is the largest ongoing prospective registry of patients with AF. The real world insights that continue to be gathered from the GARFIELD-AF registry are being converted into real-world evidence that helps inform and identify areas where the medical community can continue to improve patient outcomes.

GARFIELD-AF is a pioneering, independent academic research initiative led by an international steering committee under the auspices of the TRI, London, UK.

It is an international, observational, multicentre study of stroke prevention in patients with newly diagnosed AF. Patients were enrolled from over 1,000 centres in 35 countries worldwide, including from the Americas, Europe,Africa and Asia-Pacific.

Contemporary understanding of $\mathrm{AF}$ is based on data gathered in controlled clinical trials. Whilst essential for evaluating the efficacy and safety of new treatments, these trials are not representative of everyday clinical practice and, hence, uncertainty persists about the real-life burden and management of this disease. GARFIELD-AF seeks to provide insights into the impact of anticoagulant therapy on thromboembolic and bleeding complications seen in this patient population. It will provide a better understanding of the potential opportunities for improving care and clinical outcomes amongst a representative and diverse group of patients and across distinctive populations. This should help physicians and healthcare systems to appropriately adopt innovation to ensure the best outcomes for patients and populations.

The registry started in December 2009. Four key design features of the GARFIELD-AF protocol ensure a comprehensive and representative description of $\mathrm{AF}$; these are:

Five sequential cohorts of prospective, newly diagnosed patients, facilitating comparisons of discrete time periods and describing the evolution of treatments and outcomes;

Investigator sites that are selected randomly within carefully assigned national AF care setting distributions, ensuring that the enrolled patient population is representative;

Enrolment of consecutive eligible patients regardless of therapy to eliminate potential selection bias;

Follow-up data captured for a minimum of 2 and up to 8 years after diagnosis, to create a comprehensive database of treatment decisions and outcomes in everyday clinical practice.

Included patients must have been diagnosed with nonvalvular AF within the previous 6 weeks and have at least one risk factor for stroke; as such, they are potential candidates for anticoagulant therapy to prevent blood clots leading to stroke. It is left to the investigator to identify a patient's stroke risk factor(s), which need not be restricted to those included in established risk scores. Patients are included whether or not they receive anticoagulant therapy, so that the merit of current and future treatment strategies can be properly understood in relation to patients' individual risk profiles.

Up to $2 \%$ of the global population has $\mathrm{AF}$, including around 8.8 million people in Europe and 5-6.1 million in the United States. It is estimated that its prevalence will at least double by 2050 as the global population ages. AF is associated with a five-fold increase in stroke risk, and one out of five strokes is attributed to this arrhythmia. Ischaemic strokes related to AF are often fatal, and those patients who survive are left more frequently and more severely disabled and have a greater risk of recurrence than patients with other causes of stroke. Hence, the risk of mortality from AF-associated stroke is doubled and the cost of care is $50 \%$ higher.

AF occurs when parts of the atria emit uncoordinated electrical signals. This causes the chambers to pump too quickly and irregularly, not allowing blood to be pumped out completely. As a result, blood may pool, clot and lead to 
thrombosis, which is the number one cardiovascular killer in the world. If a blood clot leaves the left atrium, it could potentially lodge in an artery in other parts of the body, including the brain. A blood clot in an artery in the brain leads to a stroke. $92 \%$ of fatal strokes are caused by thrombosis. Stroke is a major cause of death and long-term disability worldwide - each year, 6.7 million people die and 5 million are left permanently disabled. People with AF also are at high risk for heart failure, chronic fatigue and other heart rhythm problems.

\section{FDA clearance of EnSite Precision ${ }^{\mathrm{TM}}$ cardiac mapping system and Advisor ${ }^{\mathrm{TM}}$ FL Circular Mapping Catheter, Sensor Enabled $^{\mathrm{TM}}$}

FDA clearance of its EnSite Precision ${ }^{\mathrm{TM}}$ cardiac mapping system and Advisor ${ }^{\mathrm{TM}}$ FL Circular Mapping Catheter, Sensor Enabled $^{\mathrm{TM}}$ has been announced. The new EnSite Precision cardiac mapping system is the latest addition and is designed to provide automation, flexibility and precision in cardiac mapping during the treatment of patients with abnormal heart rhythms (cardiac arrhythmias). Prominent European electrophysiology labs and hospitals have embraced this next-generation platform, which has been used in thousands of cases in Europe since receiving CE Mark in January 2016.

When physicians use catheter ablation to treat abnormal heart rhythm, a small area of heart tissue under the tip of the ablation catheter is heated by high-frequency energy, creating a lesion or tiny scar. As a result, this tissue is no longer capable of conducting or sustaining the arrhythmia. Cardiac mapping provides a live view of the heart so physicians can visualize and navigate cardiac anatomy to deliver more precise ablation therapy. The new EnSite Precision cardiac mapping system offers a new dual-technology platform that provides highly detailed anatomical models and maps to enable more efficient treatment of a wide range of arrhythmias - including complex arrhythmias, such as atrial fibrillation or ventricular tachycardia.

The EnSite Precision cardiac mapping system is based on the EnSite Velocity ${ }^{\mathrm{TM}}$ cardiac mapping system, which is utilized in more than 2,000 electrophysiology labs around the world. The EnSite Precision cardiac mapping system introduces new solutions, including the EnSite ${ }^{\mathrm{TM}}$ AutoMap Module, which will enable the electrophysiologist to more quickly perform morphology matching that identifies the source of the irregular heartbeat using automated cardiac rhythm mapping tools to facilitate treatment. The EnSite AutoMap Module includes the new TurboMap feature, which will allow the physician to build a map of the heart 10 times faster than current systems using recorded data. Researchers expect this technology to be a new standard for patients experiencing arrhythmias from multiple origins in the heart.

The EnSite Precision cardiac mapping system is flexible and enhances workflow efficiency by allowing physicians to map heart chambers with any electrophysiology catheter and customize procedures to address the circumstances of each case. The EnSite Precision cardiac mapping system and the company's sensor enabled tools also allow catheter navigation to occur with minimal fluoroscopy, thus reducing potential for risks associated with excessive radiation exposure.

The launch of the EnSite Precision cardiac mapping system includes other advancements that include new surface electrodes (patches) and sensor-enabled mapping catheters. These new tools along with the TactiCath ${ }^{\text {TM }}$ Quartz contact force ablation catheter are part of a comprehensive EP portfolio to help optimize clinical outcomes for treating arrhythmias.

\section{ENSURE-AF trial Edoxoban: a New Anticoagulant Option Before Cardioversion}

Patients with atrial fibrillation (AF) who need anticoagulation before undergoing electrical correction of their abnormal heartbeat (cardioversion) may benefit from treatment with edoxoban - a non-vitamin K antagonist (VKA) oral anticoagulant (NOAC), according to results of the ENSURE-AF trial.

While VKA anticoagulation works well, it has a major limitation in that it requires regular monitoring and dose adjustment to ensure that patients reach anticoagulation targets. This can sometimes delay cardioversion for several weeks.

Edoxaban was previously shown to be safe and effective compared to standard VKA therapy (enoxaparin/warfarin) among patients with AF in the ENGAGE AF-TIMI 48 study, but the impact of electrical cardioversion was not systematically assessed in that study.

ENSURE-AF, the largest randomized clinical trial of anticoagulation for cardioversion in patients with AF, the phase $3 \mathrm{~b}$ study, involving 239 study sites in 19 countries in Europe and the United States, included 2,199 patients with documented non-valvular AF who were scheduled for electrical cardioversion after anticoagulation therapy. 
A total of 1,095 patients were randomised to receive edoxaban, while the remaining 1,104 received enoxaparin/ warfarin (dosing varied depending on patient characteristics).

Of these $988(90 \cdot 2 \%)$ and $966(87 \cdot 5 \%)$ patients, respectively, were cardioverted either electrically or spontaneously, some with the use of transoesophageal echocardiography (TEE).

The primary efficacy objective of this study was to compare the incidences of the composite endpoint of stroke, systemic embolic event (SEE), myocardial infarction (MI) and cardiovascular (CV) death between the two groups at day 28 (ITT analysis).

This endpoint occurred at a comparable rate in both groups: $0.5 \%$ patients in the edoxaban arm vs. $1.0 \%$ in the enoxaparin/warfarin arm $(\mathrm{OR}=0 \cdot 46 ; 95 \% \mathrm{CI}, 0 \cdot 12-1 \cdot 43)$

The primary safety outcome was a composite endpoint of major and clinically relevant non-major (CRNM) bleeding events at 30 days. This also occurred at a comparable rate $1 \cdot 5 \%$ and $1 \cdot 0 \%$ respectively $(\mathrm{OR}=1 \cdot 48 ; 95 \% \mathrm{CI}, 0 \cdot 64-3 \cdot 55)$. 